\title{
Inflammation in Acute Cardiac Care
}

\section{Monica Marton-Popovici}

Department of Anesthesiology and Critical Care, Swedish Medical Center, Seattle, USA

Inflammation has been proved to represent a major player in cardiovascular diseases, being linked with various cardiac conditions. From atherosclerosis development to acute myocardial infarction, all stages of atheromatous plaque progression and vulnerabilization are characterized by an increased inflammation-mediated response. At the same time, inflammation in the post-myocardial infarction period has been demonstrated to play a significant role in the complex process of myocardial repair, scar formation, and ventricular remodeling. ${ }^{1}$ In the setting of an acute myocardial infarction, immediate and delayed inflammatory responses have been demonstrated to be in the center of several complex pathophysiological mechanisms involving scar formation, wall thinning, and left ventricular dilatation, leading to a significant decrease in the ejection fraction on long term. ${ }^{2}$

Atrial fibrillation is another example of a cardiac condition in which augmented inflammation can play a decisive role, being demonstrated that an exacerbated inflammation is directly related not only to the risk of atrial fibrillation, but also to the rate of recurrence after atrial fibrillation ablation. ${ }^{3}$ Co-existence of atherosclerosis with various diseases characterized by an increased inflammatory reaction has been described in the last years. One such example is periodontal disease, in which systemic inflammation triggered by local inflammatory reactions in the periodontal space is responsible for atherosclerosis initiation and plaque progression, finally resulting in plaque vulnerabilization and acute coronary syndrome.4,5

Increased inflammation has been demonstrated to be associated with the systemic release of various circulatory biomarkers, such as C-reactive protein (CRP), an acutephase protein produced by the liver in response to inflammatory stimuli. The high-sensitivity form of this protein, named hs-CRP, has become a routine biomarker for clinical assessment in all types of cardiovascular emergencies. ${ }^{6,7}$

Persistence of an increased inflammatory status in the post-infarction period can play a major role in the evolution of these patients. Goldstein et al. demonstrated a high number of recurrent coronary events at the 12 months follow-up in patients with AMI and complex coronary plaques. ${ }^{8}$ It is currently estimated that over $20 \%$ of patients suffering an AMI will have another major cardiovascular event in the next 12 months after the infarction. This indicates that in the case of an AMI, the vulnerable period is not limited to the immediate post-infarction phase, extending to several months after the acute event, and a careful attention should be given to attenuate the inflammatory reactions at least during the first year post-infarction.

The inflammatory response in the post-infarction period has been differentiated into a pro-inflammatory phase occurring immediately after the infarction (in the first 3 days), mainly characterized by activation of several cytokines (such as interleukin-18, interleukin-6, interferonalpha, tumor necrosis factor alpha), and a pro-reparative phase, characterized by augmented release of interleukin-10 and TGF-8, starting after 1-2 days. During these phases, a number of cells (such as neutrophils, macrophages, lymphocytes, and dendritic cells) accumulate at the site of infarcted tissue in an attempt to repair the myocardial damage. ${ }^{9}$ Inflammatory signals released by the infarcted tissue can stimulate the interaction between leukocytes and endothelial cells, promoting cell adhesion and extravasation of neutrophils and monocytes. ${ }^{2}$

Various anti-inflammatory strategies have been proposed for the prevention of left ventricular remodeling following an AMI. New drugs targeting cardiac remodeling include those that act against integrin, complement cas- 
cade, cytokines, or metalloproteases. ${ }^{10}$ The CANTOS trial was a landmark study which reshaped some fundamental concepts in cardiology, demonstrating that inhibition of the inflammatory pathway mediated by interleukin 1-beta can prevent recurrent cardiovascular events in stable post-AMI patients. The primary endpoint of CANTOS was the cumulative incidence of nonfatal AMI nonfatal stroke or CV death, the study demonstrating a significant reduction in the primary endpoint after administration of anti-inflammatory Canakinumab versus placebo, proving the role of novel cytokine-based therapy for secondary prevention of cardiovascular diseases. ${ }^{11}$ It is surprising, however, that despite of the results of the CANTOS trial, the role of anti-inflammatory therapy in the post-myocardial infarction period has not been established yet on a large scale in clinical settings.

One of the most challenging approaches dedicated to increase myocardial recovery after an ischemic episode consists in the administration of stem cells targeting the infarcted area, in the hope that paracrine effects of the stem cells would be able to attenuate the local inflammatory reactions in parallel with increasing neoangiogenesis. ${ }^{12-16}$ However, this approach remains to be validated, as major clinical trials aiming to study this hypothesis presented divergent results.

A study published in this number of JCE by Morariu et al. demonstrates a direct relationship between hs-CRP, as an inflammation-related biomarker in the immediate post-infarction period, and the extent of myocardial scar at 1 month. ${ }^{17}$ This is in line with previous reports which documented the role of inflammation in the post-infarction recovery. It should be pointed out that this study uses cardiac magnetic resonance (CMR) imaging for quantification of the myocardial scar, based on image post-processing by a dedicated software. At the same time, this study demonstrates that increased serum hs-CRP levels on day 1 post-infarction, as well as increased serum levels of interleukin- 6 are associated with larger infarct size, higher transmurality index, and worse ventricular function at CMR at 1 month post-infarction.

Currently, CMR imaging has become a routine imagistic tool for assessing cardiac function in the post-AMI period. The results of the study by Morariu et al. show us how the inflammatory reactions in acute settings can impact the cardiac status on a medium and potentially long term, providing an explanation for the findings we commonly see at the routine, image-based follow-up of patients suffering an acute cardiac event. ${ }^{17}$

\section{CONFLICT OF INTEREST}

Nothing to declare.

\section{REFERENCES}

1. Chen B, Frangogiannis NG. Immune cells in repair of the infarcted myocardium. Microcirculation. 2017;24. doi: 10.1111/ micc.12305.

2. Prahbu SD, Frangoigiannis NG. The Biological Basis for Cardiac Repair After Myocardial Infarction: From Inflammation to Fibrosis. Circ Res. 2016;119:91-112. doi: 10.1161/CIRCRESAHA.116.303577.

3. Floria M, Barboi O, Rezus C, et al. Atrial Fibrillation and GastroOesophageal Reflux Disease - Controversies and Challenges. Current Pharmaceutical Design. 2015;21:3829-3834.

4. Sincar CD, Ioanid N, Rudnic I, et al. The Biochemical Effects of Non-surgical Periodontal Therapy in Patients with and without Chronic Renal Disease. Revista de Chimie. 2017;68:605-607.

5. DeLeon-Pennell KY, Iyer RP, Ero OK, et al. Periodontalinduced chronic inflammation triggers macrophage secretion of Ccl12 to inhibit fibroblast-mediated cardiac wound healing. JCI Insight. 2017;2:94207. doi: 10.1172/jci.insight.94207.

6. Dima N, Rezus E, Singeap AM, et al. Analysis of Enzymatic Systems in the Severe Hepatic Dysfunction. Revista de Chimie. 2016;67:948-952.

7. Benedek T, Jako B, Suciu Zs, Benedek I. Correlations between severity of coronary atherosclerosis and persistent elevation of circulating C-reactive protein levels 30 days after an acute myocardial infarction. Revista Romana de Medicina de Laborator. 2014;22:49-61. doi: 10.2478/rrlm-2014-0005.

8. Goldstein JA, Demetrious D, Grines CL, Pica M, Shoukfeh M, O`Neil WW. Multiple complex coronary plaques in patients with acute myocardial infarction. N Engl J Med. 2000;343:915-922.

9. Westman PC, Lipinski MJ, Luger D, et al. Inflammation as a Driver of Adverse Left Ventricular Remodeling After Acute Myocardial Infarction. J Am Coll Cardiol. 2016;67:2050-2060. doi: 10.1016/j.jacc.2016.01.073.

10. Seropian IM, Toldo S, Van Tassel BW, Abbate A. Antiinflammatory strategies for ventricular remodeling following ST-segment elevation acute myocardial infarction. J Am Coll Cardiol. 2014;63:1593-1603. doi: 10.1016/j.jacc.2014.01.014.

11. Ridker PM, Everett BM, Thuren T, et al. Antiinflammatory Therapy with Canakinumab for Atherosclerotic Disease. N Engl J Med. 2017;377:1119-1131. doi: 10.1056/NEJMoa1707914.

12. Gyöngyösi $M$, Hemetsberger R, Wolbank S, et al. Delayed Recovery of Myocardial Blood Flow After Intracoronary Stem Cell Administration. Stem Cell Review and Reports. 2011;7:616-623.

13. Benedek I, Bucur O, Benedek T. Intracoronary Infusion of Mononuclear Bone Marrow-Derived Stem Cells is Associated with a Lower Plaque Burden After Four Years. J Atheroscler Thromb. 2014;21:217-229.

14. Gyöngyösi M, Hemetsberger R, Posa A, et al. HypoxiaInducible Factor 1-Alpha Release After Intracoronary Versus Intramyocardial Stem Cell Therapy in Myocardial Infarction. J Cardiovasc Transl Res. 2010;3:114-121. doi: 10.1007/s12265009-9154-1.

15. Lazar E, Benedek T, Korodi Sz, et al. Stem cell-derived exosomes - an emerging tool for myocardial regeneration. World J Stem Cells. 2018;10:106-115. doi: 10.4252/wjsc.v10.i8.106.

16. Tang J, Cui X, Caranasos TG, et al. Heart Repair Using NanogelEncapsulated Human Cardiac Stem Cells in Mice and Pigs with Myocardial Infarction. ACS Nano. 2017;11:9738-9749. doi: 10.1021/acsnano.7b01008.

17. Morariu M, Márton E, Mester A, Rațiu M, Benedek I. Association between Acute Inflammatory Response and Infarct Size in STEMI Patients Undergoing Primary PCI. Journal of Cardiovascular Emergencies. 2018;4:140-146. doi: 10.2478/jce-2018-0017. 